'Departamento de HematologíaOncología.

2Departamento de Cirugía Oncológica.

${ }^{3}$ Departamento de Radiología. Programa de Cáncer, Facultad de Medicina.

Pontificia Universidad Católica de Chile. Santiago, Chile. ${ }^{4}$ Hospital Sótero del Río. Santiago, Chile. Recibido el 3 de diciembre de 2014, aceptado el 8 de mayo de 2015.

Correspondencia a: César Sánchez csanchez@med.puc.cl

\section{Inmunohistoquímica convencional como predictor de respuesta y sobrevida en pacientes con cáncer de mama tratadas con quimioterapia preoperatoria. Experiencia de un centro}

\author{
FRANCISCO ACEVEDO ${ }^{1}$, MAURICIO CAMUS ${ }^{2}$, \\ CATALINA VIAL ${ }^{3}$, SERGIO PANAY ${ }^{4}$, MARCELO ABARCA ${ }^{4}$, \\ FRANCISCO DOMÍNGUEZ ${ }^{2}$, CÉSAR SÁNCHEZ $^{1}$
}

\section{Predictive value of conventional immuno- histochemical biomarkers in breast cancer}

Background: Pathological complete response ( $p C R)$ after neoadjuvant chemotherapy (NCT) in breast cancer (BC) identifies patients with good prognosis. Aim: To assess if the clinico-pathological subtype, determined by classic immunohistochemical (IHC) markers, is able to predict $p C R$ and prognosis in BC patients treated with NCT. Material and Methods: One hundred thirty three BC patients aged 24-80 years, were treated with NCT. Clinico-pathological subtype was defined based on classic IHC markers. $p C R$ was defined as the absence of invasive neoplastic cells in the breast and lymph nodes, on final breast surgery. Results: $p C R$ was achieved in $8.2 \%$ of patients, 3.5 and $19.5 \%$ in luminal and hormonal receptor $(H R)$ negative tumors respectively $(p<0.01)$. Median follow-up was 72.6 months (3.5-190). Patients who achieved $p C R$ had higher overall survival (OS) $(p=0.04)$. A univariate analysis revealed that size of the tumor, ratio of metastatic to examined lymph nodes and absence of $H R$ were significant predictors of $p C R$. These findings were not replicated in the multivariate analyses. Conclusions: Clinico-pathological subtypes were independent prognostic factors for $P C R$ and $O S$ in $B C$ patients in our cohort. These findings support using classic and cheap biomarkers as a predictive tool for NCT in BC.

(Rev Med Chile 2015; 143: 724-732)

Key words: Chemotherapy, Adjuvant; Biological markers; Breast neoplasms; Prognosis.
$\mathrm{E}$ 1 cáncer de mama $(\mathrm{CM})$ es la principal causa de muerte por cáncer en mujeres chilenas ${ }^{1}$. La quimioterapia neoadyuvante (QTN), es decir, previo a la cirugía, es una estrategia de tratamiento aceptada para pacientes con CM localmente avanzado. Este enfoque de tratamiento aumenta las posibilidades de lograr un tratamiento conservador y mejora las tasas de resecabilidad de tumores considerados inicialmente no resecables ${ }^{2}$, permitiendo la evaluación de factores pronósticos $\mathrm{y}$ predictivos, de manera dinámica e in vivo. El alcanzar una RPC posterior a QTN ha sido capaz de predecir pronóstico a largo plazo en varios estudios, y por tanto, es un potencial marcador sustituto de supervivencia ${ }^{3}$; especialmente para tumores considerados triple negativos (TN) o aquellos que sobre-expresan el receptor del factor de crecimiento epidérmico tipo 2 (HER-2). 
El estudio de perfiles genéticos ha permitido determinar los subtipos moleculares de CM. Estos permiten predecir respuesta al tratamiento y evolución de enfermedad ${ }^{4}$. La probabilidad de alcanzar RPC está directamente asociada a subtipos moleculares ${ }^{5}$. Después de QTN, tumores considerados basaloides (la mayoría TN) o HER-2 positivos logran mayor RPC que aquellos considerados luminales (estrógenos dependientes). Este último factor, la obtención de RPC, se asocia a mejor sobrevida, que aquellos que no alcanzan tal respuesta $^{6,7}$. Sin embargo, la implementación de estos perfiles genéticos en la práctica clínica tiene limitaciones, sobre todo en nuestro país, por su alto costo. Por tanto, herramientas patológicas convencionales, como el grado histológico $(\mathrm{GH})$, expresión de $\mathrm{RH}$ y expresión de HER2 pueden ser utilizadas como sustitutos de clasificación de subtipos.

El objetivo de nuestro estudio es investigar si estas herramientas de patología convencionales son capaces de predecir la respuesta a QTN en pacientes chilenas con CM, y determinar si el alcanzar RPC se asocia a mejor pronóstico.

\section{Pacientes y Métodos}

\section{Población}

Estudio retrospectivo realizado en el Centro del Cáncer de la Pontificia Universidad Católica de Chile en Santiago, Chile. El estudio fue aprobado por el Comité de Ética local.

Se reclutaron todas las pacientes con diagnóstico de CM que recibieron QTN en nuestro centro entre 1997 y 2012. Los criterios de inclusión fueron: 1) recibir al menos un ciclo de tratamiento citotóxico;2) disponibilidad de información clínica y patológica suficiente para determinar tipo de respuesta alcanzada. Los pacientes con metástasis al momento del diagnóstico se incluyeron en el análisis de la respuesta, pero se excluyeron del análisis de supervivencia.

\section{Revisión patológica}

El tipo histológico, tamaño tumoral, $\mathrm{GH}^{8}$, y el compromiso linfonodal fueron determinados en todas las muestras. Combinamos GH 1 y 2 siendo considerados como tumores con baja tasa de proliferación. El estado del receptor de estrógeno (RE), receptor de progesterona (RP) y HER2 fue determinado a través de IHQ. El valor de corte para determinar la positividad de RE y RP fue de $\geq 1 \%$ de células tumorales con tinción nuclear. Consideramos como HER-2 positivos todos aquellos tumores con puntuación de 3 cruces $(+++)$ en IHQ. En caso de ser considerados 2 cruces $(++)$, se realizó estudio de hibridación fluorescente in situ (FISH) para HER2, procedimiento realizado en la mayoría de los casos (este estudio no era obligatorio en nuestro centro hasta el año 2006). Dado que el estudio con Ki67 no se realiza de forma rutinaria en nuestro centro, decidimos no incluirlo en el análisis.

El estadio al momento del diagnóstico se determinó de acuerdo con el sistema TNM del American Joint Committee on Cancer Staging Manual 2010 (séptima edición).

\section{Subtipos clínico-patológicos}

Los tumores se clasificaron en 4 subtipos de acuerdo a marcadores clásicos: Luminal A, luminal B, HER-2 enriquecidos y TN (Tabla 1).

\section{Respuesta tumoral}

RPC fue definida como la ausencia de tumor invasor en la mama (independiente de la presencia de componente residual in situ) y en los linfonodos axilares (ypT0/is-ypN0). Pacientes con respuesta parcial o sin respuesta fueron clasificados como "no-RPC". Debido a que la definición exacta de RPC se encuentra aún en discusión, exploramos una segunda definición (RPC-2) definida como

Tabla 1. Definición de subtipos moleculares de cáncer de mama basados en inmunohistoquímica convencional y grado histológico

\begin{tabular}{|ll|}
\hline $\begin{array}{l}\text { Subtipo } \\
\text { molecular }\end{array}$ & Definición \\
\hline Luminal A & RE y/o RP $(+)$; HER-2 $(-)$; GH 1-2 \\
Luminal B & $\begin{array}{l}\text { RE y/o RP }(+) \text {; HER-2 (-) asociado } \\
\text { a: GH3 o HER-2 }(+) \text {, independiente } \\
\text { de GH }\end{array}$ \\
HER-2 enriquecido & $\begin{array}{l}\text { RE y PR }(-) \text {; HER-2 }(+) \text {, independen- } \\
\text { diente de GH }\end{array}$ \\
Triple negativo & $\begin{array}{l}\text { ER, PR and HER-2 (-), independen- } \\
\text { diente de GH }\end{array}$ \\
\hline
\end{tabular}

RE: receptor de estrógeno; RP: receptor de progesterona; HER-2: Factor de crecimiento epidérmico humano tipo 2; $\mathrm{GH}$ : grado histológico. 
la ausencia de componente invasor en la mama independiente del estado de los linfonodos (ypT0/ is-ypN0/+).

\section{Análisis estadístico}

Se utilizó $\chi^{2}$ (o prueba exacta de Fisher) para evaluar variables categóricas. Usamos prueba de Wilcoxon para comparar medianas. Realizamos un análisis multivariado para RPC a través de una regresión logística binaria. La SG fue calculada de acuerdo al método de Kaplan-Meier y comparadas mediante prueba de Log-Rank. Consideramos una diferencia estadísticamente significativa cuando el valor de $\mathrm{p}$ fue menor o igual a 0,05 . Todos los datos fueron analizados utilizando la versión de $\mathrm{IBM}^{\circledR}{ }^{\circledR} \mathrm{SPSS}^{\circledR} 21$.

\section{Resultados}

De 1.560 pacientes con CM invasor tratados en nuestro centro desde 1999 hasta 2012, determinamos 147 pacientes que recibieron QTN $(9,5 \%)$. No consideramos catorce de estos pacientes por encontrarse aún bajo tratamiento al momento del análisis ( 8 pacientes) o debido a que no fue posible reunir información adecuada (6 pacientes). Las características y distribución de los subtipos clínico-patológicos de los 133 pacientes incluidos, en comparación con el grupo control (pacientes con CM que no recibieron QTN) se presentan en la Tabla 2.

La mediana de edad de pacientes que recibieron QTN fue 51 años (24-80). La mediana del

Tabla 2. Características clínico-patológicas de pacientes con cáncer de mama que recibieron quimioterapia neoadyuvante (QTN) comparadas con el grupo control (sin QTN)

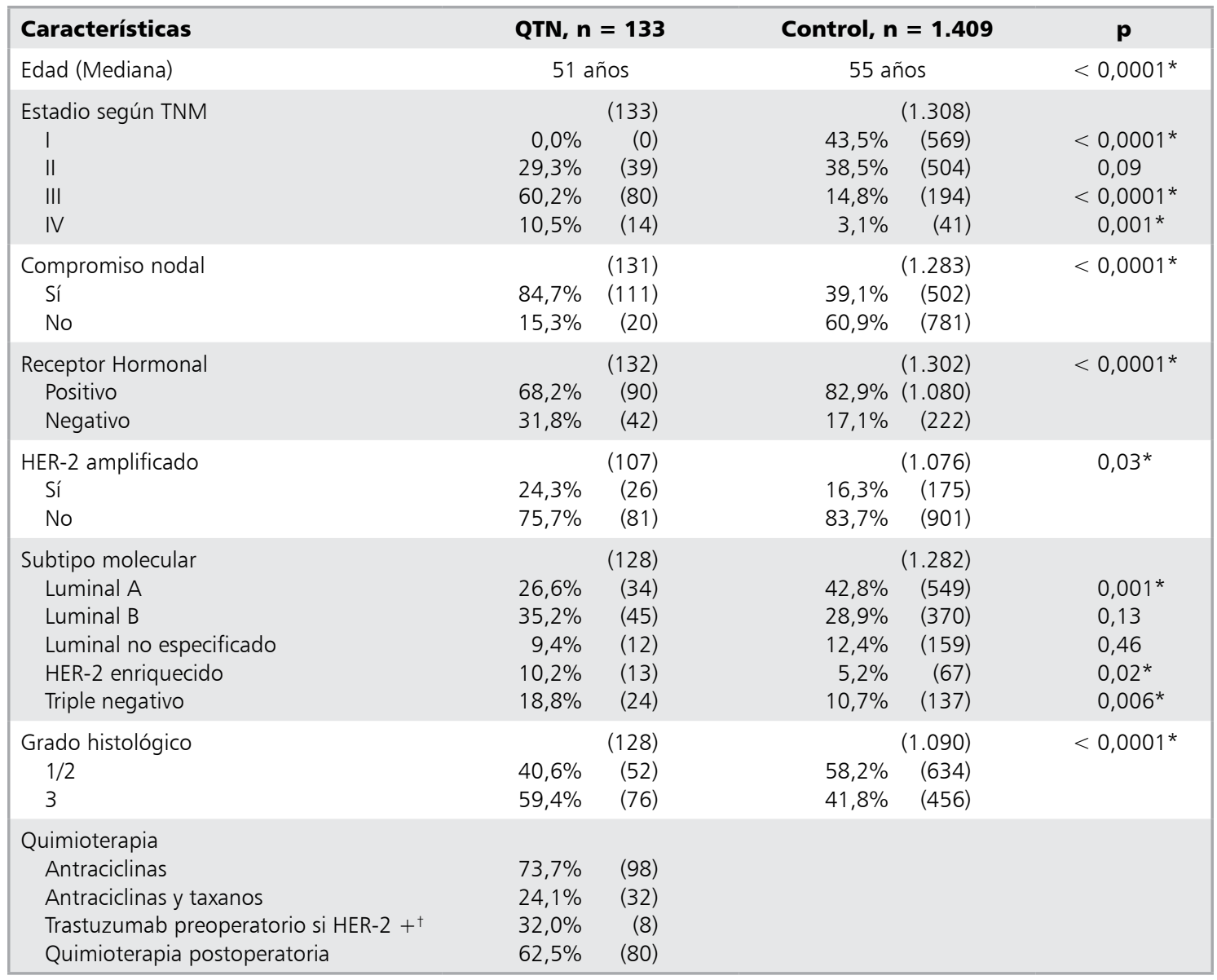

* Diferencia es estadísticamente significativa. †Tanto en Luminal B HER-2 + como en el subtipo HER-2 enriquecido. 
tamaño tumoral al examen físico fue de $6,4 \mathrm{~cm}$ (2-20). Quince pacientes (11,2\%) tenían enfermedad diseminada (estadio IV) al momento del diagnóstico. 84,3\% de los tumores correspondió a histología ductal. $55,2 \%$ de los pacientes se sometió a mastectomía total y 89,6\% fueron tratados con disección axilar.

En comparación con el grupo control, las pacientes que recibieron QTN eran más jóvenes, $(\mathrm{p}<0,0001)$, con tumores de mayor $\mathrm{GH}(56,0 \%$ vs $31,7 \%, p<0,0001)$, más frecuentemente $\mathrm{RH}$ negativos $(31,8$ vs $17,1 \%$; $\mathrm{p}<0,0001)$ y HER-2 sobre-expresado $(24,3$ vs $16,3 \%, \mathrm{p}=0,003)$.

En relación al subtipo de CM (Tabla 2) 68,9\% fueron clasificados como luminales (luminal A 35 casos, luminal B 44 casos y 12 casos indefinidos).

Casi un tercio de los tumores estudiados presentaron RH negativos, la mayoría de ellos TN ( 24 pacientes o $18,4 \%$ del total) y 13 o $10,2 \%$ del total de pacientes fueron considerados como HER-2 enriquecidos.

Las pacientes fueron tratadas con diferentes esquemas de QT: 97,7\% de ellas recibieron antraciclinas y $26,0 \%$ taxanos. $62,5 \%$ de los esquemas de QT fueron completados después del procedimiento quirúrgico.

Documentamos un total de 11 RPC $(8,2 \%)$ (Tabla 3). La tasa de RPC fue de 3,5\% en tumores que expresaban $\mathrm{RH}$ y $19,5 \%$ en aquellos que no lo expresaban $(\mathrm{p}=0,01)$. Según subtipo molecular, la tasa RPC fue variable: $0,0 \%$ en luminal A, $6,6 \%$ en luminal B, $15,4 \%$ en HER-2 enriquecido y $25,0 \%$ en tumores considerados TN $(p=0,03)$ (Tabla 4$)$. De los 3 casos de RPC en el subtipo luminal B, 2 sobre-expresaron HER-2. En el restante no logramos obtener información de HER-2.

Según el GH, la probabilidad de lograr una RPC fue de $11,8 \%$ para neoplasias GH 3 vs 3,8\%

Tabla 3. Características clínico-patológicas de pacientes con cáncer de mama que recibieron quimioterapia neoadyuvante (QTN) según respuesta patológica completa (RPC)

\begin{tabular}{|c|c|c|c|}
\hline Características & RPC (11) & No RPC (122) & $\mathbf{p}$ \\
\hline Edad (mediana) & $43(34-65)$ & $51 \quad(24-80)$ & 0,26 \\
\hline Histología & (11) & $(122)$ & 0,13 \\
\hline Ductal & $100 \% \quad(11)$ & $82,8 \%(101)$ & \\
\hline Otro & $0 \% \quad(0)$ & $17,2 \% \quad(21)$ & \\
\hline Estadio (TNM) & $(11)$ & $(122)$ & 0,30 \\
\hline ॥ & $45,5 \% \quad(5)$ & $27,9 \% \quad(34)$ & \\
\hline III & $54,5 \%(6)$ & $60,7 \% \quad(74)$ & \\
\hline IV & $0,0 \% \quad(0)$ & $11,5 \% \quad(14)$ & \\
\hline Receptor hormonal & (11) & $(121)$ & $0,004^{*}$ \\
\hline Positivo & $27,3 \% \quad(3)$ & $72,7 \% \quad(88)$ & \\
\hline Negativo & $72,7 \% \quad(8)$ & $27,3 \% \quad$ (33) & \\
\hline HER-2 & $(10)$ & (97) & 0,22 \\
\hline Positivo & $40,0 \% \quad(4)$ & $22,7 \% \quad(22)$ & \\
\hline Negativo & $60,0 \% \quad(6)$ & $77,3 \% \quad(75)$ & \\
\hline Subtipo molecular & (11) & $(117)$ & $0,04^{*}$ \\
\hline Luminal A & $0,0 \% \quad(0)$ & $29,1 \% \quad(34)$ & 0,56 \\
\hline Luminal B & $27,3 \% \quad(3)$ & $35,9 \% \quad(42)$ & 0,59 \\
\hline Luminal no especificado & $0,0 \% \quad(0)$ & $10,3 \% \quad(12)$ & 0,35 \\
\hline HER-2-enriquecido & $18,2 \% \quad(2)$ & $9,4 \% \quad(11)$ & $0,006^{*}$ \\
\hline Triple negativo & $54,5 \% \quad(6)$ & $15,4 \%$ & \\
\hline Grado histológico & (11) & $(117)$ & 0,11 \\
\hline $1 / 2$ & $18,2 \% \quad(2)$ & $42,7 \% \quad(50)$ & \\
\hline 3 & $81,8 \% \quad(9)$ & $57,3 \% \quad(67)$ & \\
\hline
\end{tabular}

*Diferencia es estadisticamente significativa. 
para tumores con baja tasa de proliferación. Esta diferencia no fue estadísticamente significativa $(\mathrm{p}=0,11)$.

Quince casos $(11,2 \%)$ de RPC fueron documentados de acuerdo con la segunda definición (RCP-2). Ninguna de las características estudiadas

\section{Tabla 4. Tasa de respuesta patológica completa (RPC) después de quimioterapia neoadyuvante (QTN) según subtipo}

\begin{tabular}{|lc|}
\hline Subtipo & RPC \\
\hline Luminal A & $0,0 \%(0)$ \\
\hline Luminal B & $6,6 \%(3)$ \\
\hline Luminal B HER-2 (-) & $0,0 \%(0)$ \\
\hline Luminal B HER-2 (+) & $15,4 \%(2)$ \\
\hline HER-2-enriquecido & $15,4 \%(2)$ \\
\hline Triple Negativo & $25,0 \%(6)$ \\
\hline
\end{tabular}

y mencionadas previamente varió significativamente entre ambas definiciones (Tabla 5).

En el análisis univariado, teniendo en cuenta características como: edad, motivo de consulta, tamaño del componente invasor, tipo histológico, estadio clínico TNM, número de linfonodos resecados, proporción de metástasis a linfonodos versus linfonodos examinados, la expresión de $\mathrm{RH}$, HER-2, GH y el tipo de QT; los factores asociados con RPC fueron: el tamaño del componente invasor $(\mathrm{p}=0,004)$; la relación de metástasis a linfonodos versus linfonodos examinados $(\mathrm{p}=0,003)$; y la ausencia de RH $(p=0,009)$. Ninguno de estos factores mantuvo su significación estadística en el análisis multivariado.

Con una mediana de seguimiento de 72,6 meses (3,5 a 180 meses), la SG a 5 años fue de $100 \%$ en aquellos pacientes que lograron RPC y de $81 \%$ en aquellos que no lograron tal respuesta $(\mathrm{p}=0,037)$ (Figura 1).

Tabla 5. Características clínico-patológicas de pacientes con cáncer de mama que recibieron quimioterapia neoadyuvante (QTN) según respuesta patológica completa (RPC-2)

\begin{tabular}{|c|c|c|c|}
\hline Características & RPC-2 (15) & NO RPC-2 (118) & $\mathbf{p}$ \\
\hline Histología & (15) & $(118)$ & 0,07 \\
\hline Ductal & $100 \% \quad(11)$ & $82,2 \% \quad(97)$ & \\
\hline Otro & $0 \% \quad(0)$ & $17,8 \% \quad(21)$ & \\
\hline Estadio según TNM & (15) & (118) & 0,84 \\
\hline$\|$ & $33,3 \% \quad(5)$ & $28,8 \% \quad(34)$ & \\
\hline III & $60,0 \% \quad(9)$ & $60,2 \% \quad(71)$ & \\
\hline IV & $6,7 \% \quad(1)$ & $11,0 \% \quad(13)$ & \\
\hline Receptor hormonal & (15) & $(117)$ & $<0,0001^{*}$ \\
\hline Positivo & $26,7 \% \quad(4)$ & $74,4 \% \quad(87)$ & \\
\hline Negativo & $73,3 \%(11)$ & $25,6 \% \quad(30)$ & \\
\hline HER-2 & (14) & (93) & 0,28 \\
\hline Positivo & $35,7 \% \quad(5)$ & $22,6 \% \quad(21)$ & \\
\hline Negativo & $64,3 \% \quad(9)$ & $77,4 \% \quad(72)$ & \\
\hline Subtipo molecular & (15) & (113) & \\
\hline Luminal A & $0,0 \% \quad(0)$ & $30,1 \% \quad(34)$ & $0,009 *$ \\
\hline Luminal B & $26,7 \% \quad(4)$ & $36,3 \% \quad(41)$ & 0,46 \\
\hline Luminal no especificado & $0,0 \% \quad(0)$ & $10,6 \% \quad(12)$ & 0,36 \\
\hline HER-2-enriquecido & $20,0 \% \quad(3)$ & $8,8 \% \quad(10)$ & 0,18 \\
\hline Triple negativo & $53,3 \% \quad(8)$ & $14,2 \% \quad(16)$ & $0,001 *$ \\
\hline Grado histológico & (15) & $(113)$ & 0,08 \\
\hline $1 / 2$ & $20,0 \% \quad(3)$ & $43,4 \% \quad(49)$ & \\
\hline 3 & $80,0 \%(12)$ & $56,6 \% \quad(64)$ & \\
\hline
\end{tabular}

*Diferencia estadísticamente significativa. 


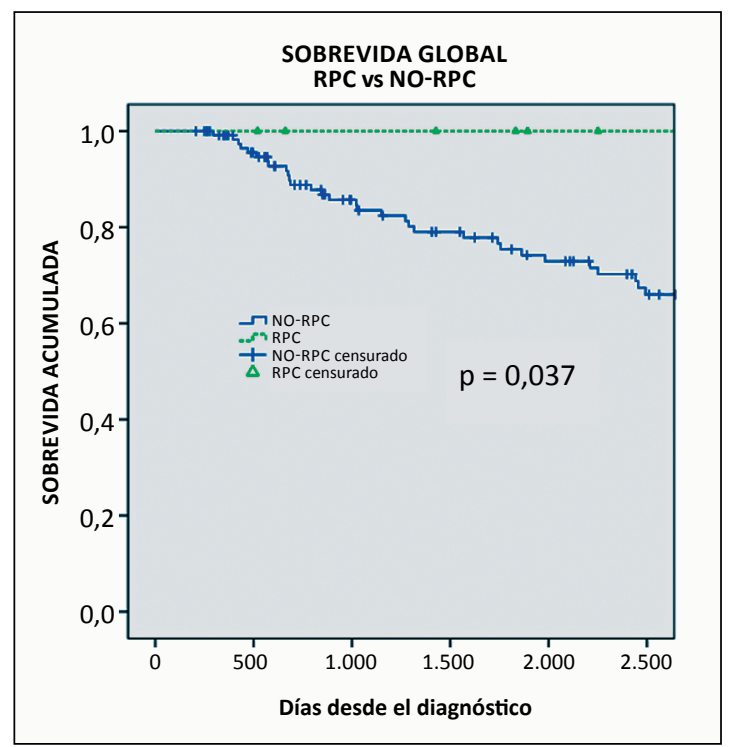

Figura 1. Curva de Kaplan Meier comparando sobrevida global en pacientes con cáncer de mama que reciben quimioterapia neoadyuvante según presencia o ausencia de respuesta patológica completa (RPC o No-RPC respectivamente).

\section{Discusión}

El CM es una enfermedad biológicamente muy heterogénea. Perou et al. ${ }^{4}$ lograron describir cuatro clases moleculares de CM, en base a expresión de perfiles genéticos distintos, los cuales fueron llamados "subtipos intrínsecos". Ellos fueron asociados inicialmente con pronóstico en CM; sin embargo, también ha sido sugerido su rol en predecir respuesta a tratamiento .

El tratamiento neoadyuvante permite la evaluación in vivo de la sensibilidad a la QT. Subtipos moleculares de CM muestran diferentes sensibilidades a la QTN, y el grado de respuesta patológica alcanzado se asocia con SG, particularmente en tumores considerados como basaloides y HER-2 enriquecidos $^{3,7}$.

¿Cómo podemos identificar estos subtipos en la práctica clínica? Se han desarrollado varios modelos utilizando distintas firmas genéticas ${ }^{10-12}$. Una de las más ampliamente utilizadas es Oncotype $D X$, el cual calcula un puntaje otorgando riesgo de recurrencia basada en expresión de 16 genes $^{10}$. Estos genes están relacionados con la expresión del RE, HER2 y varios genes asociados con proliferación. Si bien esta prueba es ampliamente utilizada en la toma de decisiones clínicas en países desarrollados, múltiples restricciones técnicas y económicas limitan su uso en la práctica clínica habitual en pacientes con CM en nuestro país.

Si bien algunos autores han sugerido que la utilización de estos test debiese representar el "gold-standard"13, la evidencia sugiere que variables clínicas y patológicas convencionales logran proporcionar información pronóstica independiente de la puntuación otorgada por marcadores moleculares ${ }^{14}$. Según el Consenso Internacional de Expertos de St. Gallen, si no es posible la identificación de los subtipos intrínsecos mediante el uso de marcadores moleculares-genéticos (por ejemplo: Oncotype DX o Mammaprint), se recomienda definiciones sustitutas utilizando la IHQ estándar9.

Sin embargo, la asociación de marcadores patológicos convencionales (ER, PR, HER-2) con los subtipos intrínsecos a través de estudios moleculares no es perfecta ${ }^{15}$.

La medición de la expresión de Ki67 permite adicionar mayor información ${ }^{16}$. Ki67 es una proteína nuclear expresada por las células en distintas fases del ciclo celular (fase tardía de G1, S y en G2/M), reflejando la proporción de células que se encuentran en proliferación en ese momento. Un Ki67 elevado se asocia a peor pronóstico y parece ser un factor predictor independiente de respuesta a QTN ${ }^{17,18}$. Recientemente, se ha demostrado que el uso de un panel de marcadores de IHQ que considere RE, RP, HER-2 y Ki67, es capaz de otorgar información pronostica similar al Oncotype $\mathrm{DX}^{14}$, apoyando el uso de IHQ habitual como sustituto a firmas de expresión genética.

Sin embargo, el uso rutinario de Ki67 para evaluación pronóstica en CM no se considera como una práctica estándar. Su costo y la falta de estandarización en la medición limitan su uso, no siendo parte del informe patológico habitual en todos los centros ${ }^{19}$. Un panel de expertos de la Sociedad Americana de Oncología Clínica (ASCO) sugiere no utilizar marcadores de proliferación para evaluar pronóstico del $\mathrm{CM}^{20}$. Otros marcadores de proliferación celular informados rutinariamente en la biopsia de mama como el GH y el índice mitótico (IM) han demostrado correlación con Ki67 en algunos estudios, sin embargo, este hallazgo no logró ser replicado en nuestro grupo ${ }^{21,22}$. Por otra parte, según Lips et $\mathrm{al}^{23}$; el uso del GH supera al uso de Ki67 como marcador sustituto al momento de definir subtipos moleculares. Esta observación 
puede estar relacionada con el hecho de que $\mathrm{GH}$ toma en consideración otras características del tumor, además de la actividad proliferativa (principal rol del Ki-67), tales como el grado de formación de glándulas y el polimorfismo nuclear, lo cual, teóricamente, podría estar relacionado con mayor sensibilidad a terapia citotóxica ${ }^{23}$.

Dado que los subtipos clínico-patológicos, como hemos discutido, presentan una buena concordancia con perfiles genéticos demostrando un papel pronóstico y predictivo valioso, decidimos evaluar la correlación entre la expresión de marcadores clásicos (RH, HER2, GH, excluyendo Ki67) y el grado de respuesta a QTN en nuestros pacientes con CM.

Los resultados de nuestro estudio mostraron una tasa global de RPC de 8,2\% posterior a QTN. Este valor parece menor a lo reportado en estudios previos ${ }^{24,25}$ lo cual puede ser explicado por múltiples factores.

Primero, en esos estudios la definición de RPC fue menos estricta a la utilizada por nosotros. Tanto en el estudio NSABP B-18 y el NSABP B-27 aquellas pacientes que sólo recibían antraciclinas como QTN lograban tasas de RPC de 13\% y $13,7 \%$, respectivamente. La definición utilizada en ambos ensayos fue la ausencia de componente invasor sólo en el tumor primario, independiente de lo que ocurría a nivel linfonodal. Estos resultados son muy cercanos a $11,2 \%$ obtenido en nuestro centro al utilizar la misma definición (RPC-2). Segundo, múltiples estudios han demostrado que incorporar un taxano antes de la cirugía se asocia con aumento de $\mathrm{RPC}^{24,26,27}$. En nuestro grupo, $62,5 \%$ de las pacientes completó el tratamiento planificado después de la cirugía y sólo $25 \%$ de ellas recibió taxanos como parte de la QTN. Por último, hay pruebas que demuestran que pacientes con enfermedad HER-2 positiva logran mayor tasa de RPC si el tratamiento incluye un agente dirigido contra HER-2, como el anticuerpo monoclonal trastuzumab ${ }^{25}$. En nuestra serie, menos de un tercio de los pacientes que sobre-expresaban HER-2, recibieron trastuzumab preoperatorio.

En el análisis univariado también encontramos que el tamaño del componente invasivo ( $\mathrm{p}$ $=0,004)$, la relación linfonodos metastásicos y examinados $(\mathrm{p}=0,003)$ y la ausencia de $\mathrm{RH}(\mathrm{p}$ $=0,009)$ fueron potenciales predictores de RPC. Ambos, el tamaño del tumor y el compromiso nodal son potentes factores pronósticos en $\mathrm{CM}^{28}$.
A pesar de que el tamaño tumoral se correlaciona con el compromiso linfonodal, los valores pronósticos de ambos factores son independientes. En algunos estudios, la proporción de linfonodos positivos extirpados es capaz de predecir sobrevida de manera acertada ${ }^{29}$. Desafortunadamente ninguno de dichos factores mantuvo su significancia luego del análisis multivariado.

En nuestro trabajo, como ha sido reportado anteriormente, pacientes que logran RPC tienen mejor $\mathrm{SG}^{3}$. Sin embargo, no todos los estudios han demostrado los mismos resultados ${ }^{24}$. Si bien esta discrepancia puede deberse a limitaciones metodológicas y al tipo de paciente incluido, von Minckwitz et al, mostró recientemente que la definición de RPC es fundamental ${ }^{3}$. En base a su trabajo, los únicos pacientes que debiesen ser considerados como RPC son aquellos que no presentan ningún tipo de tumor (in situ o invasor) en mama y axila posterior a QTN. Puesto que objetivamos pocos eventos que cumpliesen esa definición ( 8 casos) decidimos no incluirlo en nuestro análisis. Sin embargo, el uso de otras definiciones, tal como hemos mencionado anteriormente, también se asocia a pronóstico ${ }^{24}$.

Es importante destacar que estas herramientas permiten la clasificación en subtipos, identificando pacientes que con mayor probabilidad lograrán desaparición del tumor después de QTN. Esto se aplica particularmente para tumores definidos como TN y HER2-enriquecidos, con patrones de recurrencia temprana, y en el que la RPC es un marcador pronóstico aceptado y validado.

Nuestros hallazgos refuerzan el valor de la clasificación de subtipos de CM utilizando técnicas de IHQ disponibles en cualquier laboratorio de patología convencional, lo que permite predecir respuesta patológica tras QTN y además SG.

\section{Referencias}

1. Berry DA, Cronin KA, Plevritis SK, Fryback DG, Clarke L, Zelen M, et al. Effect of screening and adjuvant therapy on mortality from breast cancer. N Engl J Med 2005; 353: 1784-92.

2. Kaufmann M, Hortobagyi GN, Goldhirsch A, Scholl S, Makris A, Valagussa P, et al. Recommendations from an international expert panel on the use of neoadjuvant (primary) systemic treatment of operable breast cancer: an update. J Clin Oncol 2006; 24: 1940-9. 
3. Von Minckwitz G, Untch M, Blohmer J-U, Costa SD, Eidtmann H, Fasching PA, et al. Definition and impact of pathologic complete response on prognosis after neoadjuvant chemotherapy in various intrinsic breast cancer subtypes. J Clin Oncol 2012; 30: 1796-804.

4. Perou CM, Sørlie T, Eisen MB, van de Rijn M, Jeffrey SS, Rees CA, et al. Molecular portraits of human breast tumours. Nature 2000; 406: 747-52.

5. Rouzier R, Perou CM, Symmans WF, Ibrahim N, Cristofanilli M, Anderson K, et al. Breast cancer molecular subtypes respond differently to preoperative chemotherapy. Clin Cancer Res 2005; 11: 5678-85.

6. Untch M, Fasching PA, Konecny GE, Hasmüller S, Lebeau A, Kreienberg R, et al. Pathologic complete response after neoadjuvant chemotherapy plus trastuzumab predicts favorable survival in human epidermal growth factor receptor 2-overexpressing breast cancer: results from the TECHNO trial of the AGO and GBG study groups. J Clin Oncol 2011; 29: 3351-7.

7. Liedtke C, Mazouni C, Hess KR, André F, Tordai A, Mejia JA, et al. Response to neoadjuvant therapy and long-term survival in patients with triple-negative breast cancer. J Clin Oncol 2008; 26: 1275-81.

8. Elston CW, Ellis IO. Pathological prognostic factors in breast cancer. I. The value of histological grade in breast cancer: experience from a large study with long-term follow up. Histopathology 1991; 19: 403-10.

9. Goldhirsch A, Winer EP, Coates AS, Gelber RD, Piccart-Gebhart M, Thürlimann B, et al. Personalizing the treatment of women with early breast cancer: highlights of the St Gallen International Expert Consensus on the Primary Therapy of Early Breast Cancer 2013. Ann Oncol 2013; 2206-23.

10. Paik S, Shak S, Tang G, Kim C, Baker J, Cronin M, et al. A multigene assay to predict recurrence of tamoxifen-treated, node-negative breast cancer. N Engl J Med 2004; 351: 2817-26.

11. van't Veer LJ, Dai H, van de Vijver MJ, He YD, Hart AA, Mao M, et al. Gene expression profilling predicts clinical outcome of breast cancer. Nature 2002; 415: 530-6.

12. Parker JS, Mullins M, Cheang MCU, Leung S, Voduc D, Vickery T, et al. Supervised risk predictor of breast cancer based on intrinsic subtypes. J Clin Oncol 2009; 27: 1160-7.

13. Nielsen TO, Parker JS, Leung S, Voduc D, Ebbert M, Vickery $\mathrm{T}$, et al. A comparison of PAM50 intrinsic subtyping with immunohistochemistry and clinical prognostic factors in tamoxifen-treated estrogen receptor-positive breast cancer. Clin Cancer Res 2010; 16: 5222-32.
14. Cuzick J, Dowsett M, Pineda S, Wale C, Salter J, Quinn E, et al. Prognostic value of a combined estrogen receptor, progesterone receptor, Ki-67, and human epidermal growth factor receptor 2 immunohistochemical score and comparison with the Genomic Health recurrence score in early breast cancer. J Clin Oncol 2011; 29: 4273 8.

15. Prat A, Perou CM. Deconstructing the molecular portraits of breast cancer. Mol Oncol. Elsevier B.V. 2011; 5: 5-23.

16. Cheang MCU, Chia SK, Voduc D, Gao D, Leung S, Snider J, et al. Ki67 index, HER2 status, and prognosis of patients with luminal B breast cancer. J Natl Cancer Inst 2009; 101: 736-50.

17. Brown JR, Digiovanna MP, Killelea B, Lannin DR, Rimm DL. Quantitative assessment Ki-67 score for prediction of response to neoadjuvant chemotherapy in breast cancer. Lab Invest. Nature Publishing Group 2013; 00: 1-9.

18. Nishimura R, Osako T, Okumura Y, Hayashi M, Arima N. Clinical significance of Ki-67 in neoadjuvant chemotherapy for primary breast cancer as a predictor for chemosensitivity and for prognosis. Breast Cancer 2010; 17: 269-75.

19. Polley M-YC, Leung SCY, McShane LM, Gao D, Hugh JC, Mastropasqua MG, et al. An International Ki67 Reproducibility Study. J Natl Cancer Inst 2013; 1-10.

20. Harris L, Fritsche H, Mennel R, Norton L, Ravdin P, Taube S, et al. American Society of Clinical Oncology 2007 update of recommendations for the use of tumor markers in breast cancer. J Clin Oncol 2007; 25: 5287 312.

21. Galea MH, Blamey RW, Elston CE, Ellis IO. The Nottingham Prognostic Index in primary breast cancer. Breast Cancer Res Treat 1992; 22: 207-19.

22. Rakha EA, Reis-Filho JS, Baehner F, Dabbs DJ, Decker T, Eusebi V, et al. Breast cancer prognostic classification in the molecular era: the role of histological grade. Breast Cancer Res 2010; 12: 207.

23. Lips EH, Mulder L, de Ronde JJ, Mandjes IAM, Koolen BB, Wessels LFA, et al. Breast cancer subtyping by immunohistochemistry and histological grade outperforms breast cancer intrinsic subtypes in predicting neoadjuvant chemotherapy response. Breast Cancer Res Treat 2013; 140: 63-71.

24. Rastogi P, Anderson SJ, Bear HD, Geyer CE, Kahlenberg MS, Robidoux A, et al. Preoperative chemotherapy: updates of National Surgical Adjuvant Breast and Bowel Project Protocols B-18 and B-27. J Clin Oncol 2008; 26: 778-85.

25. Esserman LJ, Berry DA, DeMichele A, Carey L, Davis 
SE, Buxton $\mathrm{M}$, et al. Pathologic complete response predicts recurrence-free survival more effectively by cancer subset: results from the I-SPY 1 TRIAL-CALGB 150007/150012, ACRIN 6657. J Clin Oncol 2012; 30: 3242-9.

26. Bear HD, Anderson S, Smith RE, Geyer CE, Mamounas EP, Fisher B, et al. Sequential preoperative or postoperative docetaxel added to preoperative doxorubicin plus cyclophosphamide for operable breast cancer: National Surgical Adjuvant Breast and Bowel Project Protocol B-27. J Clin Oncol 2006; 24: 2019-27.

27. Smith IC, Heys SD, Hutcheon AW, Miller ID, Payne S,
Gilbert FJ, et al. Neoadjuvant chemotherapy in breast cancer: significantly enhanced response with docetaxel. J Clin Oncol 2002; 20: 1456-66.

28. Atkinson EN, Brown BW, Montague ED. Tumor volume, nodal status, and metastasis in breast cancer in women. J Natl Cancer Inst 1986; 76: 171-8.

29. Hatoum HA, Jamali FR, El-Saghir NS, Musallam KM, Seoud M, Dimassi H, et al. Ratio between positive lymph nodes and total excised axillary lymph nodes as an independent prognostic factor for overall survival in patients with nonmetastatic lymph node-positive breast cancer. Ann Surg Oncol 2009; 16: 3388-95. 